\title{
Analytical model for the transmission of electromagnetic waves through arrays of slits in perfect conductors and lossy metal screens
}

\author{
Rui Yang, ${ }^{1, a)}$ Raúl Rodríguez-Berral, ${ }^{2, b)}$ Francisco Medina, ${ }^{3, c)}$ and Yang Hao ${ }^{4, d)}$ \\ ${ }^{1}$ National Laboratory of Antennas and Microwave Technology, Xidian University Xi' an 710071, China \\ ${ }^{2}$ Department of Applied Physics 1, University of Seville, 41012-Seville, Spain \\ ${ }^{3}$ Department of Electronics and Electromagnetism, University of Seville, 41012-Seville, Spain \\ ${ }^{4}$ School of Electronic Engineering and Computer Science, Queen Mary University of London, London E1 4NS, \\ United Kingdom
}

(Received 3 October 2010; accepted 28 March 2011; published online 17 May 2011)

\begin{abstract}
This paper presents a very simple analytical model for the analysis of the resonant transmission of microwaves or millimeter waves through periodically distributed slits in a thick metal screen. The model is based on equivalent circuits consisting of transmission line elements of known characteristic admittances and propagation constants loaded by capacitors. Closed-form analytical expressions are provided for all the circuit parameters. Alternatively, the circuit parameters can be quickly computed from numerical simulations carried out at a few frequency points. The proposed analytical model accounts for all the details of the observed transmission spectrum, including conventional Fabry-Pérot (FP) resonances, which are controlled by the thickness of the screen, as well as extraordinary transmission peaks, which are related to the periodicity. The range of validity of the model as a function of dimensional parameters is discussed. The experimentally observed and numerically predicted redshift of the Fabry-Pérot transmission peaks with respect to the ideal Fabry-Pérot resonance condition is accurately accounted for by the capacitors of the model. For narrow slits, the extraordinary transmission peak is linked to the singular behavior of the capacitances at the Rayleigh-Wood anomaly frequency point. Finally, the effect of the lossy nature of the metal screens is included in the model, providing accurate predictions of the transmission losses. Additionally, for lossy screens the model adequately predicts the anomalous behavior of the above mentioned redshift when the slit width becomes comparable to the skin depth in the metal, which is in good agreement with experimental and theoretical data previously reported for a single slit. (C) 2011 American Institute of Physics. [doi:10.1063/1.3583561]
\end{abstract}

\section{INTRODUCTION}

The study of the extraordinary transmission (ET) of electromagnetic waves through electrically small apertures in conducting screens has been a hot topic since the publication of the seminal work by Ebbesen and co-workers. ${ }^{1}$ Scientific challenges and promising applications have justified such interest. Presently, it can be accepted that this is a mature area of research for which authoritative reviews are available to the interested reader. ${ }^{2-5}$ Most of this research has been focused on the study of 2-D periodic arrays of finite size holes made in metal screens. However, the case of 1-D periodic distributions of infinitely long slits (diffraction grating) deserves specific attention. This was the first system considered with the purpose of providing sound theoretical explanations for ET. ${ }^{6}$ Actually, the behavior of metal strip gratings has been a topic of interest for decades, and ET through arrays of electrically narrow slits made in a thick perfect conductor was reported as early as $1967 .^{7}$ More recently, much attention has been paid to the behavior of the

\footnotetext{
${ }^{a)}$ Electronic mail: rui.yang@elec.qmul.ac.uk. Present address: School of Electronic Engineering and Computer Science, Queen Mary University of London, London E1 4NS, United Kingdom.

${ }^{\text {b)} E l e c t r o n i c ~ m a i l: ~ r r b e r r a l @ u s . e s . ~}$

${ }^{c)}$ Electronic mail: medina@us.es.

${ }^{d)}$ Electronic mail: yang.hao@elec.qmul.ac.uk.
}

transmission spectrum of slit gratings under various conditions. For instance, multiple Fabry-Pérot internal resonances are obtained for thick screens. ${ }^{8,9}$ Also, unexpectedly high transmission (extraordinary transmission) has been observed for operation close to the Rayleigh-Wood (RW) anomaly frequency in the case of thin screens. ${ }^{6}$ Moreover, complex spectra with deep and narrowband notches (phase resonances) in the middle of the transmission bands have been reported for compound transmission gratings. ${ }^{10,11}$ In addition to periodic arrays of slits, the study of the transmission through a single slit aperture made in a flat metal screen has also been a subject of interest. ${ }^{12-14}$ From the analysis of this simple structure it is possible to qualitatively understand some important details of the behavior of more complex periodic geometries. Single slits in metal screens with periodic corrugations around them ${ }^{15}$ or electrically small dielectric scatterers in their proximity ${ }^{16,17}$ have also recently been studied.

This paper focuses on the behavior of periodic distributions of simple slits with the purpose of developing analytical solutions or fast methods to obtain the transmission/reflection spectra. Some phenomena initially reported for a single slit will also be studied in the context of the infinite periodic slit grating system, where similar qualitative behavior is expected but quantitative results are considerably different.

The peaks and dips appearing in the transmission spectra through periodic structures have been related to the existence 
of surface plasmon polaritons (SPPs) supported by the periodically shaped conducting surface. Some authors have also reported on ET in the absence of this kind of surface wave, ${ }^{18}$ although a different type of surface wave is still involved in the case studied. However, different studies reported on ET through small diaphragms located inside closed metallic waveguides, ${ }^{19-22}$ which showed that ET is not always linked to the presence of surface waves. This fact motivated some of the authors of this paper to carry out efforts to develop a unified theoretical framework for a variety of situations found in practice. The model is based on a methodology commonly used in microwave engineering. It must be emphasized that, for periodic structures, the interpretation of the transmission peaks in terms of the interaction of the impinging wave with SPPs gives an accurate picture of the phenomenon, but even in those cases it fails to provide us with detailed qualitative and quantitative information a priori, besides the fact that it is usually not the most straightforward approach to the problem. Thus, for instance, the exhaustive knowledge of the dispersion curves for the involved SPP waves is not required if one is solely interested, as usual, in the transmission and reflection coefficients for the impinging plane wave. These coefficients can be directly obtained without intermediary surface wave excitation and transmission peaks, and they can be qualitatively anticipated using the impedance matching concept. This point of view, implicit to some extent in the waveguide model described in Ref. 23, was first developed in detail in Ref. 24. The explanation of ET in Ref. 24 is consistent with the theoretical results reported in Refs. 19 and 20 for small diaphragms inside hollow pipe waveguides. In addition, it also explains conventional (large holes) and extraordinary (small holes) transmission peaks through periodic structures. Thus, the theory in Ref. 24 automatically accounts for both SPP-mediated processes and for cases where SPPs do not play any role. ${ }^{21,22}$ The impedance matching approach lends itself to simple equivalent circuits having a small number of parameters to be determined. Once these parameters are known, the transmission and reflection spectra in the frequency band of interest can be analytically obtained. This fact constitutes a remarkable advantage over fully numerical approaches.

The study in Ref. 24 was restricted to 2-D arrays of holes made in perfect conductors. However, as discussed previously, 1-D diffraction gratings are also interesting and still attract the attention of researchers. ${ }^{25-27}$ These gratings do not behave exactly as 2-D arrays of holes since the slits support propagating modes which are absent inside small holes. ${ }^{6}$ The extension of the theory in Ref. 24 to simple and compound 1-D slit gratings has recently been published by some of the authors. ${ }^{28}$ However, the model for the simple strip grating was introduced in Ref. 28 in a rather heuristic manner. Moreover, no analytical formulas were reported for some of the relevant parameters and the strengths and weaknesses of the model were not appropriately discussed. In contrast, the present paper provides an in-depth analysis of the features of the circuit model, including rigorous analytical (instead of heuristic) support. Moreover, closed-form expressions are now provided for all the parameters of the circuit model, thus yielding a fully analytical, very simple, and accurate description of the behavior of slit gratings. We will also show how the range of validity of the model can be extended by using an alternative scheme in which the circuit parameters are numerically obtained from the scattering parameters (computed using a full-wave numerical approach) at a few frequency points.

From the numerical and analytical results, it is remarkable that some of the features of the transmission spectrum of a single slit also appear in diffraction gratings, although the quantitative results are very different. For instance, the redshift of the Fabry-Pérot resonances with respect to the theoretical value obtained from the thickness of the metal screen is a phenomenon occurring in both periodic ${ }^{8,9}$ and isolated single slit $^{12-14}$ systems. However the quantitative value of the redshift is quite different in both situations. We will show how our analytical model accurately accounts for this fact in a very simple manner.

A final and significant improvement with respect to the previous model ${ }^{28}$ consists of the extension of the equivalent circuit to account for ohmic losses in the screen. This is achieved by introducing a very simple modification in the model developed for the lossless case. With this extended model, the power absorption and the slight modification of the maximum transmission frequency associated with losses can be analytically anticipated. An interesting effect of losses is that the redshift mentioned in the previous paragraph is shown to exhibit an anomalous behavior when the slit width is extremely small and becomes comparable to the skin depth in the conducting material. This qualitative behavior was reported some years ago for a single slit structure. $^{29}$ This anomaly consists of the decrease of the resonance frequency when decreasing the slit width, in contrast with the monotonous increase observed for perfect conductors. Once again, our model accurately reproduces this subtle phenomenon. Finally, it is worth mentioning that similar analytical models can be used to deal with other wave phenomena, such as extraordinary transmission of acoustic waves. ${ }^{30}$

\section{EQUIVALENT CIRCUIT FOR A PERIODIC ARRAY OF SLITS IN A THICK METAL SCREEN}

Let us consider a periodic array of identical slits made in a thick conducting plate. Figure 1(a) represents a lateral view of the system under study, with $d$ being the period of the structure, $h$ the thickness of the metal plate, and $a$ the slit width. A linearly polarized transverse electromagnetic (TEM) wave impinges normally on the structure with its magnetic field oriented parallel to the slits ( $p$-polarization). The scattering parameters for the periodic infinite structure are identical to those of the waveguide discontinuity problem shown in Figs. 1(b)-1(c) (Ref. 28) (a similar waveguide discontinuity approach has been previously applied to 2-D arrays of circular or rectangular holes ${ }^{23,24}$ ). Note that $W$ can be arbitrarily chosen since the fields do not depend on the $x$ variable. In this way, the problem has been reduced to the computation of the scattering parameters of a height-step discontinuity in a parallel plate waveguide. This problem is easily amenable to the mode matching solution ${ }^{31}$ (a modal approach applied to slit systems can also be found, for 


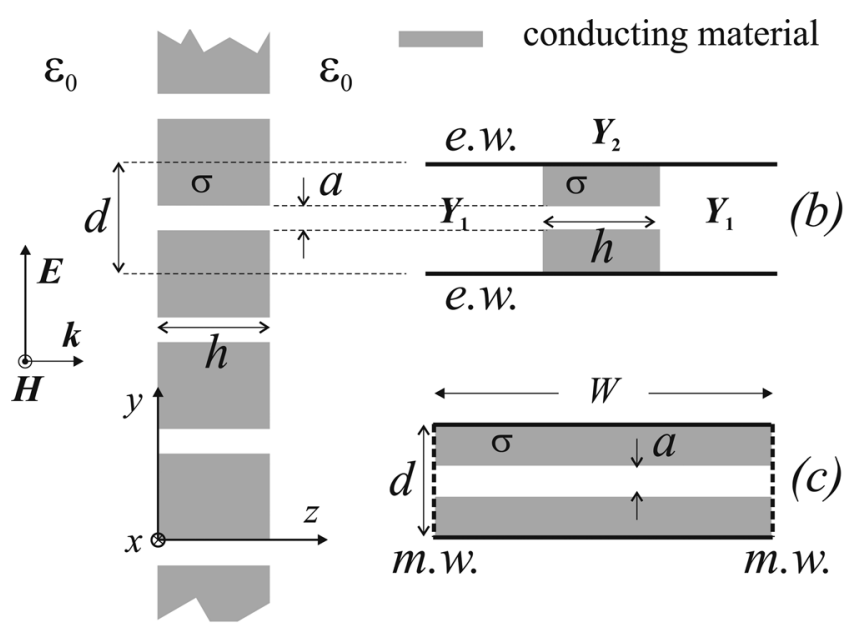

(a)

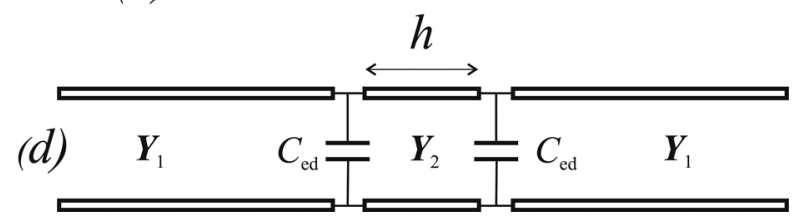

FIG. 1. (a) Cross section of the slit grating considered in this paper (the structure is uniform along the $x$ direction). (b) and (c) show the lateral and frontal views of the unit cell valid for normal incidence. (d) Equivalent circuit for the unit cell problem. The conductivity can be infinite to deal with the perfect conductor case. (Note: e.w. represents electric wall, and m.w. represents magnetic wall).

instance, in Ref. 10). In most of the cases treated in the literature, the wavelength of the impinging field is taken to be longer than $d$ in order to enforce a single transmitted and reflected spot (nondiffracting operation regime). In other words, only the TEM mode propagates along the two different waveguide sections in Fig. 1(b). All of the higher order waveguide modes required to match the boundary conditions at the discontinuity planes [all of them transverse magnetic (TM) modes $^{28}$ ] operate below cut-off. Since we are interested in the scattering coefficients for the fundamental TEM mode, an equivalent transmission line circuit $^{32}$ such as the one shown in Fig. 1(d) is proposed to account for the physical situation. Next, we will demonstrate that this equivalent circuit is rigorous in the narrow slit limit $(a \ll d)$, and we will show how the different high-order waveguide modes contribute to the shunt capacitors.

Let us first consider the perfect conductor case $(\sigma \rightarrow \infty)$. The structure in Fig. 1(b) corresponds to two cascaded height-step discontinuities such as that shown in Fig. 2(a). The electromagnetic field at both sides of the discontinuity can be written as a superposition of waveguide modes. The transverse (to $z$ ) components of the fields at the discontinuity plane $(z=0)$ can be expressed as a combination of propagating and evanescent modes of the waveguide with height $d$, namely

$$
\begin{gathered}
E_{1}(y)=E_{1,0}^{+}+E_{1,0}^{-}+\sum_{n=1}^{\infty} E_{1, n}^{-} \cos \left(k_{1, n} y\right), \\
H_{1}(y)=\xi_{1,0}\left(E_{1,0}^{+}-E_{1,0}^{-}\right)-\sum_{n=1}^{\infty} \xi_{1, n} E_{1, n}^{-} \cos \left(k_{1, n} y\right),
\end{gathered}
$$

(a)

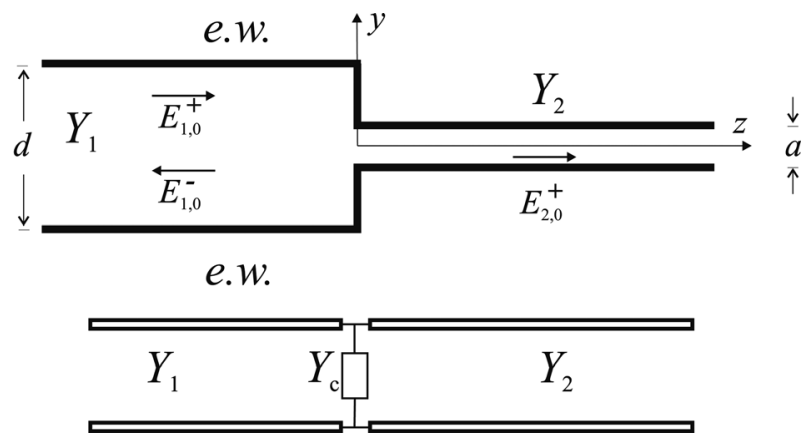

FIG. 2. (a) Longitudinal section of the height step discontinuity under study. (b) Equivalent circuit model for the structure depicted in (a).

where the superscript $+(-)$ corresponds to waves propagating toward increasing (decreasing) values of $z$. The first subscript (1) represents the region where the fields are expanded (region 1 for $z<0$ ). The second subscript represents the order of the mode ( $n=0$ for the propagating TEM mode and $n>1$ for the remaining below-cutoff TM modes). The transverse wavenumber in region 1 is $k_{1, n}=(2 n \pi) / d$ (note that a virtual electric wall exists at $y=0)$. Finally, $\xi_{1, n}$ are the wave admittances of the different modes at the left-hand side of the height step discontinuity. Of course, a similar expansion can be done using the modes at the right-hand side parallel plate waveguide

$$
\begin{gathered}
E_{2}(y)=E_{2,0}^{+}+\sum_{n=1}^{\infty} E_{2, n}^{+} \cos \left(k_{2, n} y\right), \\
H_{2}(y)=\xi_{2,0} E_{2,0}^{+}+\sum_{n=1}^{\infty} \xi_{2, n} E_{2, n}^{+} \cos \left(k_{2, n} y\right),
\end{gathered}
$$

with $k_{2, n}=(2 n \pi) / a$. The wave admittances are given by

$$
\xi_{i, n}=\mathrm{j} \frac{\xi_{i, 0}}{\sqrt{\left(f_{i, n}^{c} / f\right)^{2}-1}},
$$

where $f_{i, n}^{c}$ is the cutoff frequency of mode $n$ in region $i$. Note that, for the TEM mode,

$$
\xi_{1,0}=\xi_{2,0}=\sqrt{\varepsilon_{0} / \mu_{0}}
$$

since we are assuming that the slit is filled with air. Nevertheless, the corresponding value of $\xi_{2,0}$ could be used if a different dielectric material is filling the slit region. The generalized scattering matrices of the height-step discontinuity can be obtained by enforcing the continuity of the fields at the $z=0$ plane and projecting over the modal transverse field profiles [i.e., the cosines in Eqs. (1) and (2)]. This approach is usually referred to as mode matching, and it will be used later on as an alternative numerical strategy to compute the circuit parameters.

From the analytical point of view, we are interested in the study of mode matching equations under the narrow slit approximation, since this analysis will lead us to our circuit model. From Eq. (1a) we have 


$$
\int_{d / 2}^{d / 2} E_{1}(y) \cos \left(k_{1, n} y\right) d y=\left\{\begin{array}{ll}
d\left(E_{1,0}^{+}+E_{1,0}^{-}\right) & n=0 \\
\frac{d}{2} E_{1, n}^{-} & n \geq 1
\end{array} .\right.
$$

For $a \ll d$ the higher order modes inside the narrow waveguide can be neglected, in such a way that only the TEM fields in Eq. (2) are retained. Thus, applying the continuity of the fields for $|y| \leq a / 2$ and taking into account that the tangential electric field vanishes on the perfect conducting screen we obtain

$$
d\left(E_{1,0}^{+}+E_{1,0}^{-}\right)=a E_{2,0}^{+} .
$$

The perfect conductor (zero tangential electric field) condition at the screen also implies that

$$
\int_{d / 2}^{d / 2} E_{1}(y) \cos \left(k_{1, n} y\right) d y=\int_{a / 2}^{a / 2} E_{1}(y) \cos \left(k_{1, n} y\right) d y,
$$

and taking into account that $\cos \left(k_{1, n} y\right) \approx 1$ over the interval $-a / 2 \leq y \leq+a / 2$, provided that $n$ is significantly smaller than $d / a$, we can write

$$
E_{1, n}^{-} \approx 2\left(E_{1,0}^{+}+E_{1,0}^{-}\right),
$$

i.e., the amplitudes of the higher order modes are independent of their order. This is what one would expect from the Fourier series expansion of a Dirac delta function, which is a good approximation for the electric field distribution along $y$ (at the $z=0$ plane) when the slit is extremely narrow. This fact allows us to introduce drastic simplifications in the equations, leading to our transmission line circuit model. Indeed, from Eq. (1b) and the simplified version of Eq. (2b) (i.e., the version that ignores higher order modes) and enforcing the continuity of the magnetic field we obtain

$$
\xi_{1,0}\left(E_{1,0}^{+}-E_{1,0}^{-}\right)-\sum_{n=1}^{\infty} \xi_{1, n} E_{1, n}^{-} \cos \left(k_{1, n} y\right)=\xi_{2,0} E_{2,0}^{+} .
$$

Now combining Eqs. (6), (8), and (9)

$$
\begin{aligned}
& {\left[\xi_{1,0}-\frac{d}{a} \xi_{2,0}-\sum_{n=1}^{\infty}\left(2 \xi_{1, n}\right)\right] E_{1,0}^{+}} \\
& \quad=\left[\xi_{1,0}+\frac{d}{a} \xi_{2,0}+\sum_{n=1}^{\infty}\left(2 \xi_{1, n}\right)\right] E_{1,0}^{-} .
\end{aligned}
$$

If the concept of characteristic admittance of the parallel plates waveguides involved in our problem is introduced, Eq. (10) can be rewritten in a fashion that clearly resembles the reflection coefficient $(\Gamma)$ of the voltage waves in the transmission line circuit depicted in Fig. 2(b), which is given by

$$
\Gamma=\frac{E_{1,0}^{-}}{E_{1,0}^{+}}=\frac{V_{1}^{-}}{V_{1}^{+}}=\frac{Y_{1}-\left[Y_{2}+Y_{C}\right]}{Y_{1}+\left[Y_{2}+Y_{C}\right]} .
$$

By comparison of Eqs. (10) and (11), $Y_{1}=(W / d) \xi_{1,0}$ and $Y_{2}=(W / a) \xi_{2,0}$ are the characteristic admittances (as defined in conventional transmission line theory ${ }^{32}$ ) of the parallel plate transmission lines of width, $W$ (arbitrary), and heights, $d$ and $a$, respectively. The shunt admittance in Eq. (11) accounts for the effect of the evanescent modes and is given by

$$
Y_{C}=\frac{W}{d} \sum_{n=1}^{\infty} 2 \xi_{1, n}
$$

The complex reflection coefficient, $\Gamma$, is valid for the electric fields of the original electromagnetic problem and for the voltage waves of the transmission line model (note that $V_{1}^{ \pm}=d E_{1,0}^{ \pm}$). The current waves along the transmission line model are related with the magnetic fields of the TEM mode through the simple relation, $I_{1}^{ \pm}=W H_{1}^{ \pm}$. Equation (12) shows that, for $a / d \ll 1, Y_{C}$ is a sum of purely reactive admittances with a positive imaginary part (capacitive admittances). Therefore, the reactive load associated with higher order TM modes can be represented by the shunt connection of the elementary capacitors, each of them accounting for a high-order TM mode. Of course, this is rigorously correct in the limit of zero width slits $(a / d \rightarrow 0)$. In general, the capacitances of these elementary capacitors are frequency dependent, as is clearly indicated by Eq. (3). Nevertheless, for frequencies well below the cutoff of any of the higher order modes it is straightforward to show that the wave admittances in Eq. (3) are proportional to the angular frequency, $\omega$. Thus, the shunt admittance can be reduced to a single capacitor with a frequency-independent capacitance, $C_{\text {ed }}$, namely

$$
Y_{C}=\mathrm{j} \omega C_{\mathrm{ed}},
$$

(for harmonic fields having a time domain dependence of the form $\exp (\mathrm{j} \omega t)$. Obviously, the global capacitance, $C_{\text {ed }}$ represents the electrostatic edge capacitance of the $90^{\circ}$ corners in Fig. 2(a). This is expected to be a good approximation for narrow slits and frequencies well below the cutoff of the high order modes. But this very simple model naturally fails when the operation frequency is comparable to the cutoff frequency of the first high order mode (the $\mathrm{TM}_{2}$ mode), i.e., to the onset frequency of the first grating lobe $f_{c}^{\mathrm{TM}_{2}}=c / d$, where $c$ is the speed of light in vacuum. In such a case, the frequency dependence of the capacitance associated to the $\mathrm{TM}_{2}$ mode cannot be neglected (indeed, it diverges for $f \rightarrow f_{c}^{\mathrm{TM}_{2}}$ ). Nevertheless, the frequency dependence of this capacitance is known from Eq. (3) and therefore its contribution to the global edge capacitance can be explicitly taken into account by splitting the edge capacitance into two terms as

$$
C_{\text {ed }}(f)=C_{0}+\frac{C_{\mathrm{TM}_{2}}}{\sqrt{1-\left(f / f_{c}^{\mathrm{TM}_{2}}\right)^{2}}},
$$

where $C_{0}$ represents the contribution of the remaining high order modes and where both $C_{0}$ and $C_{\mathrm{TM}_{2}}$ are independent of frequency.

Finally, the scattering parameters of the structure can be easily obtained from a conventional transmission line analy$\mathrm{sis}^{32}$ of the complete model in Fig. 1(d). The symmetry of the structure with respect to the middle plane of the transmission line length representing the slit (the one with characteristic 
admittance, $Y_{2}$, see Fig. 1) allows us to define the reflection coefficients for even excitation, $S_{11}^{\mathrm{e}}$, and for odd excitation, $S_{11}^{o}$ (see Ref. 28):

$$
\begin{gathered}
S_{11}^{\mathrm{e}}=\frac{Y_{1}-\mathrm{j}\left[\omega C_{\text {ed }}+Y_{2} \tan (\theta / 2)\right]}{Y_{1}+\mathrm{j}\left[\omega C_{\text {ed }}+Y_{2} \tan (\theta / 2)\right]} \\
S_{11}^{\mathrm{o}}=\frac{Y_{1}-\mathrm{j}\left[\omega C_{\text {ed }}-Y_{2} \cot (\theta / 2)\right]}{Y_{1}+\mathrm{j}\left[\omega C_{\text {ed }}-Y_{2} \cot (\theta / 2)\right]},
\end{gathered}
$$

where $\omega$ is the angular frequency of operation and $\theta=k_{0} h$ is the electrical thickness of the screen (i.e., the electrical length of the transmission line with characteristic admittance, $\left.Y_{2}\right), k_{0}$ being the wavenumber in vacuum $\left(k_{0}=\omega / c\right)$. The reflection $\left(S_{11}\right)$ and transmission $\left(S_{12}\right)$ coefficients of the original structure can be computed from $S_{11}^{\mathrm{e}}$ and $S_{11}^{\mathrm{o}}$ applying the superposition

$$
S_{11}=\frac{1}{2}\left(S_{11}^{\mathrm{e}}+S_{11}^{\mathrm{o}}\right) ; \quad S_{12}=\frac{1}{2}\left(S_{11}^{\mathrm{e}}-S_{11}^{\mathrm{o}}\right) .
$$

In the expressions above, the electrical length, $\theta$, is real valued provided losses are neglected. This restriction will be relaxed later to explain the behavior of slits in lossy conductors. In particular, the case of very narrow slits in lossy conductors will be considered in order to provide an explanation of the interesting anomalous behavior of resonance frequencies reported by Suckling et al. ${ }^{29}$ within the frame of our analytical model.

\section{COMPUTATION OF THE MODEL PARAMETERS}

Since the geometry of the transmission lines representing free space regions and slits is particularly simple (parallel plate waveguides), the characteristic admittances and propagation constants are known in closed form. These parameters can be obtained from the per unit length capacitances of the parallel plate lines. These capacitances are $C_{1}=\varepsilon_{0} W / d$ for the virtual transmission line representing free space and $C_{2}=\varepsilon_{0} W / a$ for the slit region. For perfect conductors, no more information is required and the characteristic admittances of the transmission lines in Fig. 1(d) would be $Y_{1}=1 /\left(c C_{1}\right)$ and $Y_{2}=1 /\left(c C_{2}\right)$. Of course, these values of the characteristic admittances are identical to those obtained from the expressions in terms of the wave admittances of the TEM modes given in the previous section. Therefore, the problem to be solved is to determine a suitable value of $C_{\text {ed }}$ and its two contributions $C_{0}$ and $C_{\mathrm{TM}_{2}}$. As already mentioned in the previous section, for frequencies well below the onset of the first higher order mode of the waveguide with height, $d$ (i.e., for frequencies well below $\left.f_{c}^{\mathrm{TM}_{2}}=c / d\right)$, the value of the global capacitance $C_{\text {ed }}$ can be obtained from electrostatic considerations. ${ }^{28}$ Fortunately, it is possible to obtain a closed form expression for the electrostatic edge capacitance of the step discontinuity by applying conformal mapping techniques. ${ }^{32}$ Thus, in the quasistatic limit we have the following expression for $C_{\mathrm{ed}}$

$$
\begin{aligned}
C_{\mathrm{ed}}(0) & =C_{0}+C_{\mathrm{TM}_{2}} \\
& =\frac{\varepsilon_{0} W}{\pi}\left[2 \ln \left(\frac{1-x^{2}}{4 x}\right)+\left(\frac{1}{x}+x\right) \ln \left(\frac{1+x}{1-x}\right)\right],
\end{aligned}
$$

where $x=a / d$.

In order to account for the behavior of the structure at frequencies close to $f_{c}^{\mathrm{TM}_{2}}$, an estimation of $C_{\mathrm{TM}_{2}}$ is required. For narrow slits, we can easily obtain an expression for this parameter from the theory presented in the previous section. Thus, from Eqs. (3), (12), and (14) we have

$$
\begin{aligned}
Y_{\mathrm{TM}_{2}}= & \mathrm{j} \omega \frac{C_{\mathrm{TM}_{2}}}{\sqrt{1-\left(f / f_{c}^{\mathrm{TM}_{2}}\right)^{2}}} \\
& \approx 2 \mathrm{j} \frac{W}{d} \frac{\xi_{1,0}}{\sqrt{\left(f_{c}^{\mathrm{TM}_{2}} / f\right)^{2}-1}}
\end{aligned}
$$

and therefore,

$$
C_{\mathrm{TM}_{2}} \approx \varepsilon_{0} W / \pi
$$

Note that for narrow slits, this contribution is independent of the width $a$. Since the overall value of $C_{\text {ed }}$ increases when $a$ decreases, it is obvious that the relative weight of the contribution of the first TM mode is larger for relatively wide slits. It will be shown later that Eq. (19) is not accurate enough for wide slits and an alternative empirical correction will be provided. The combination of the circuit model with the closedform expressions given in Eqs. (17) and (19) describes a fully analytical model for the system under study.

An alternative method to obtain $C_{0}$ and $C_{\mathrm{TM}_{2}}$ in Eqs. (17) and (19) is based on the use of numerically computed full-wave scattering parameters. A numerical code based on the mode matching scheme mentioned in the previous section has been written for this purpose. The code has been exhaustively verified by comparing with the results from the application of the commercial finite element software HFSS. In order to compute the parameters of the circuit, the global capacitance can be related to, for instance, the reflection coefficient for the even excitation case as

$$
C_{\mathrm{ed}}(f)=\frac{\mathrm{j}}{\omega}\left[\mathrm{j} Y_{2} \tan (\theta / 2)-Y_{1} \frac{1-S_{11}^{\mathrm{e}}}{1+S_{11}^{\mathrm{e}}}\right] .
$$

Taking into account this relation and the fact that $C_{\mathrm{ed}}$ depends linearly on $C_{0}$ and $C_{\mathrm{TM}_{2}}$, it is possible to use a linear least squares algorithm to extract very stable and accurate values of $C_{0}$ and $C_{\mathrm{TM}_{2}}$ from a few numerically generated values of $S_{11}^{\mathrm{e}}$. This approach has the advantage of extending, to some extent, the range of validity of the circuit model to wider slits. This is because when the slit width is relatively large, the circuit model is still able to provide accurate results if the values of $C_{0}$ and $C_{\mathrm{TM}_{2}}$ are freely adjusted from full-wave data instead of using the analytical values reported in Eqs. (17) and (19). This strategy is also interesting from a validation point of view, since it provides a method for checking the accuracy of the above analytical expressions for $C_{0}$ and $C_{\mathrm{TM}_{2}}$. Of course, we cannot expect the model to work for very large slit widths if the frequency is close to the onset of the first grating lobe. For such cases the model itself does not give an appropriate account of the physical situation, even from the qualitative point of view. Nevertheless, mode matching results would still be valid, of course. 


\section{RESULTS AND DISCUSSION}

This section includes various results for the circuit parameters of the model and for the transmission spectrum obtained from them, using both the fully analytic model and the alternative numerical approach. A comparison between the results obtained using these two approaches and the fullwave mode matching results are discussed and interpreted in connection with the material presented in the previous sections.

First, Fig. 3 shows the low-frequency total capacitance $\left(C_{0}+C_{\mathrm{TM}_{2}}\right)$ and each of the two contributions separately as functions of the width of the slit (normalized to the period). Solid lines correspond to numerically generated data (extracted from the full-wave scattering parameters obtained from mode matching) whereas dashed lines represent the values predicted by the closed-form expressions (17) and (19). The first interesting observation is that the closed-form expression given in (17) perfectly agrees with the numerically generated results for the whole range of values of the slit width shown in the figure. However, as was expected, the value of $C_{\mathrm{TM}_{2}}$ in expression (19) provides accurate results only in the narrow slit limit (say, for $a / d<0.1$ ). From the numerically extracted values, it is observed that $C_{\mathrm{TM}_{2}}$ is a decreasing function of $a / d$, since it can be qualitatively expected from the simplified mode matching procedure reported above. In order to account for the effect of the slit width on $C_{\mathrm{TM}_{2}}$, it is found that the following empirical formula (represented by the circles in Fig. 3)

$$
C_{\mathrm{TM}_{2}} \approx \frac{\varepsilon_{0} W}{\pi} \cos \left(\frac{\pi a}{d}\right)
$$

yields a good matching to numerically generated data. This is a reasonably good estimation of the contribution of the first higher order mode to the total capacitance. The accuracy of this parameter is not important for frequencies well below the onset of the first TM mode (the total quasistatic capacitance is quite accurate, and this is all we need), but it becomes critical at frequencies close to that one. Thus, we

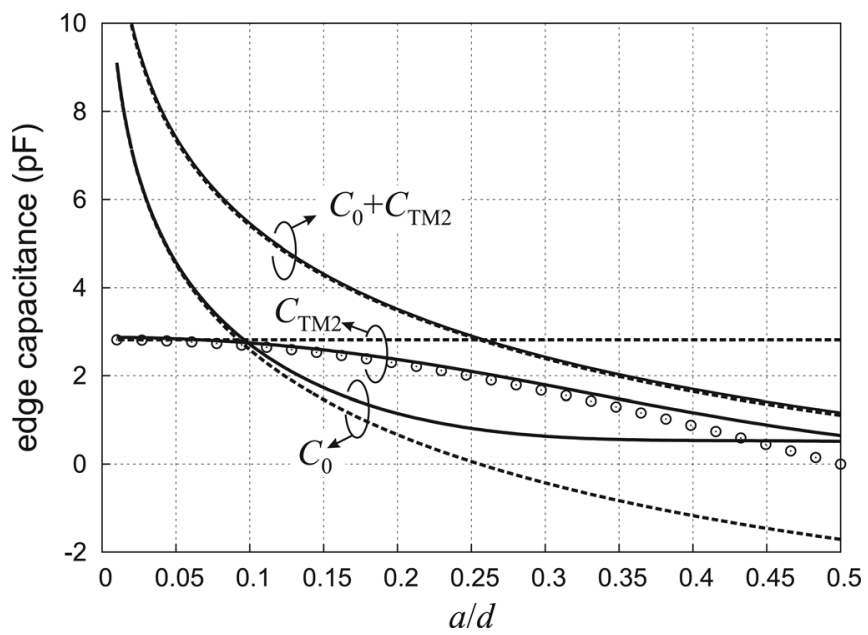

FIG. 3. Total electrostatic capacitance $\left(C_{0}+C_{\mathrm{TM}_{2}}\right)$ and partial contributions $\left(C_{\mathrm{TM}_{2}}\right.$ and $\left.C_{0}\right)$ from analytical formulas (17) and (19) (dashed lines) and from numerical data (solid lines). The circles correspond to the empirical formula for $C_{\mathrm{TM}_{2}}$ given in Eq. (21). can use Eqs. (17) and (21) together with Eq. (14) as an "improved" analytical model. It will be shown in brief that these analytical parameters provide very good results of the transmission spectrum for the range of values of a/d for which the circuit model is expected to be applicable. It can be seen in the figure that Eq. (21) loses accuracy for wide slits $(a / d>0.4)$, but this is not relevant since for such wide slits the circuit model itself is not valid.

Next, Fig. 4 shows the transmission coefficient curves versus frequency for frequencies below the onset of the first higher order mode. The solid lines correspond to full-wave data generated directly with a mode matching numerical code, which are considered to be exact. Dashed lines are obtained using the circuit model with the analytical parameters in Eqs. (17) and (21). The results are given for three different slit widths: $a / d=0.1$ (narrow slit), $a / d=0.2$ (medium-size slit), and $a / d=0.4$ (wide slit). The analytical model yields very good results for frequencies well below $f_{c}^{\mathrm{TM}_{1}}$ in all three cases, as expected. Discrepancies can be observed in the frequency range close to $f_{c}^{\mathrm{TM}_{2}}$, but only for the wide slit case. For the two narrowest slits the model captures both the FP regime (resonant frequencies far below $f_{c}^{\mathrm{TM}_{2}}$ ) and the so-called extraordinary transmission regime (a transmission peak close to $f_{c}^{\mathrm{TM}_{2}}$ which is not related with FP resonances but controlled by the period, $d$, of the structure).

A close inspection of Fig. 4 reveals that the frequency of the FP resonances (i.e., the frequency of the two first total transmission peaks) decreases as $a / d$ increases. This redshift is very similar to the one reported in Ref. 12 for the single slit case, although of different magnitude. In the context of our model, a useful interpretation of the redshift has been given $^{28}$ in terms of an equivalent thickness of the conducting screen as follows. For $a \ll d$, it is clear that $Y_{2} \gg Y_{1}$. Consequently, the original problem (1-D array of slits) can be viewed as the study of the scattering parameters of a high characteristic admittance transmission line section of length, $h$, inserted into a transmission line environment with a significantly smaller characteristic admittance. If the effect of the higher order modes diffracted at the discontinuities is neglected (i.e., if $C_{\mathrm{ed}}$ is considered to be vanishingly small), we would have an elementary transmission line Fabry-Pérot resonator having transmission resonances for those frequencies making the thickness, $h$, an integer number of free-space wavelengths, i.e., at $h=n \lambda_{0} / 2$. Typically, $C_{\text {ed }}$ can be neglected if $a \ll h$, since in this case the electromagnetic energy associated with evanescent fields is very small in comparison with the energy associated with traveling waves. However, if $h$ is only a few times that of $a$ or even smaller than $a$, the effect of $C_{\text {ed }}$ cannot be neglected at all. When the nonvanishing value of $C_{\mathrm{ed}}$ is taken into account, the resonance frequencies (transmission peaks) predicted by Eqs. (15a), (15b), and (16) are given by the following rigorous equation, $^{28}$

$$
\tan (\theta)=-\frac{2 \omega Y_{2} C_{\mathrm{ed}}}{Y_{2}^{2}-Y_{1}^{2}-\left(\omega C_{\mathrm{ed}}\right)^{2}} .
$$

For very narrow slits the right-hand side of Eq. (22) vanishes and therefore $\theta_{\text {res }}=n \pi / 2$. Otherwise, Eq. (22) predicts a 
redshift similar to the one reported in Ref. 12 for the single slit case, although of different magnitude. Thus, the actual FP resonance frequencies (transmission peaks) would correspond to an enlarged ideal FP transmission line resonator having a length, $h+\Delta h$, where the excess length, $\Delta h$, can be seen as the extension of the slit region required to incorporate the influence of $C_{\text {ed }}$ into the transmission line itself. This extra thickness would then be $\Delta h=2 C_{\text {ed }} / C_{2}$. A transmission line section of length, $h+\Delta h$, terminated by perfect magnetic walls would resonate at the same frequencies as the original transmission line section loaded with the capacitors. It should be mentioned that this interpretation requires that $Y_{2}$ is significantly larger than $Y_{1}$ and $h$ is not too small in comparison with $a$. But even for those cases the circuit model predictions of Eq. (22) are valid.

As shown by Fig. 4, the fully analytical circuit model cannot accurately account for the region of extraordinary transmission in the case of wide slits. However, if the parameters $C_{0}$ and $C_{\mathrm{TM}_{2}}$ are extracted from a few frequency points using the least squares method, it is possible to account even for that extreme case. Since the differences between analytical formulas and numerically derived parameters can only be appreciated in the region around the extraordinary transmission peak, let us focus our attention on that frequency range. In Fig. 5(a) we plot the mode matching (exact) results together with the analytical values obtained with the formulas in (17) and (21). Similar data are shown in Fig. 5(b), but now the capacitances are extracted from the numerical scattering parameters computed at a few frequency samples using a least squares algorithm. It is clear that the numerically extracted values of $C_{0}$ abd $C_{\mathrm{TM}_{2}}$ yield a better agreement with the mode matching results, thus extending the applicability of the model. From these results one might think that this strategy provides good results for any value of $a / d$, which we must emphasize is not correct. The circuit model is qualitatively wrong in the region of frequencies close to $f_{c}^{\mathrm{TM}_{2}}$ for wide enough slits. For instance, we can see that, due to the divergence of the $C_{\mathrm{TM}_{2}}$ capacitance, the proposed circuit model predicts a transmission zero at $f=f_{c}^{\mathrm{TM}_{2}}$ (the socalled Rayleigh-Wood anomaly frequency). However, close inspection of the curve for $a / d=0.4$ in Fig. 5(b) shows that some transmission occurs at that frequency point (the numerically computed value for $\left|S_{21}\right|$ at $f=f_{c}^{\mathrm{TM}_{2}}$ is approximately, 0.1). The transmissivity at $f=f_{c}^{T M_{2}}$ increases when $a / d$ increases, and this fact is not taken into account by our model. Perhaps a different circuit model should be used for slit widths larger than about $50 \%$ of the period. However, for narrower slits our model yields surprisingly accurate results.

A final point deserves some attention. In our model we have neglected the interaction between the two height step discontinuities through higher order modes. Roughly speaking, this is reasonable for screen thicknesses larger than $a$. We have studied the case of very thin conducting screens using the numerical approach to obtain the circuit parameters (note that in the case of very thin conducting screens only the extraordinary transmission peaks occur). In order to account for the interaction through higher order modes we have developed two circuit models, one for even excitation and the other for odd excitation, having different edge
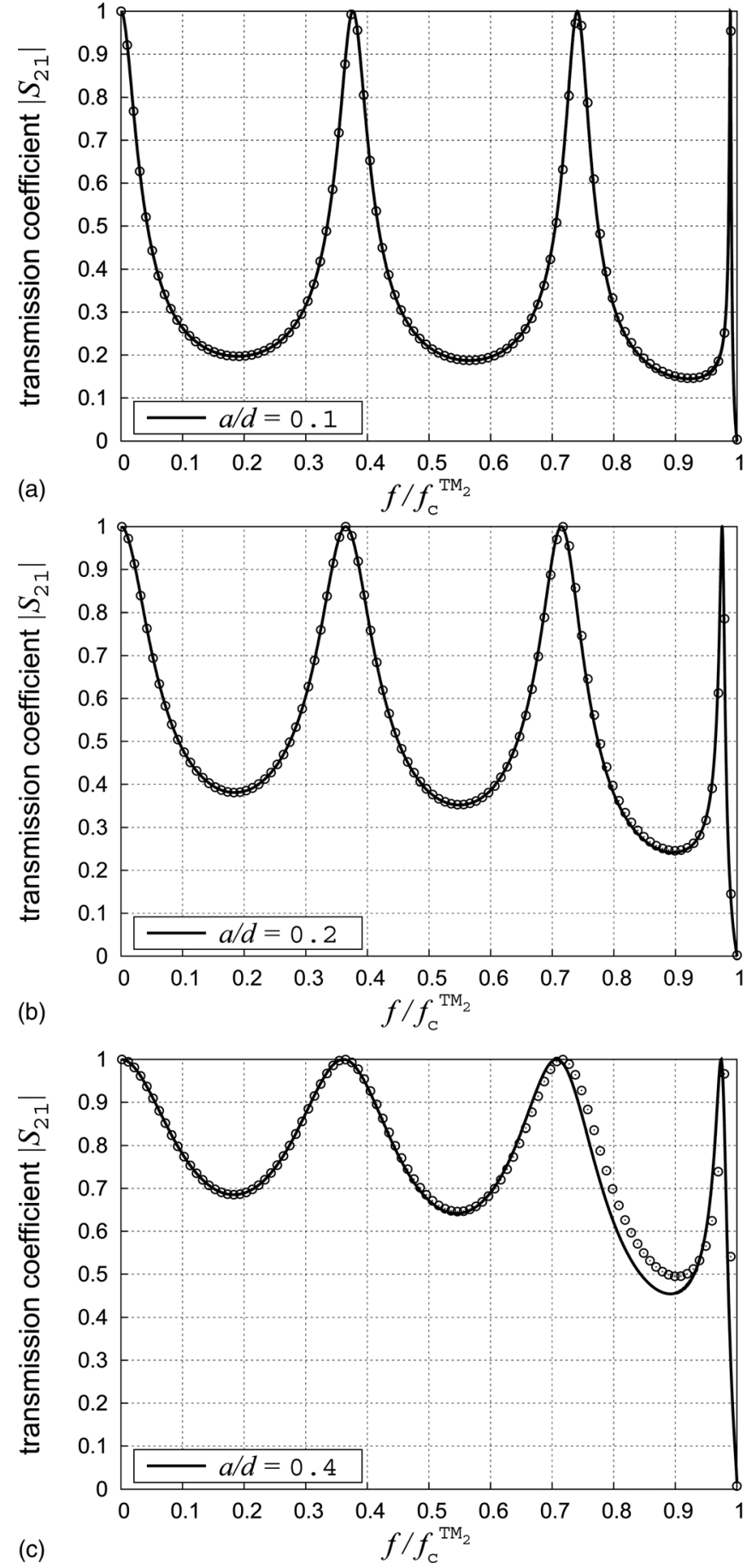

FIG. 4. Comparison between mode matching and analytical data for three different slit widths: (a) narrow slit, $a / d=0.1$; (b) medium width slit, $a / d=0.2$, and (c) wide slit, $a / d=0.4$. The screen thickness is $h / d=1.2$ for all three cases.

capacitances. The edge capacitances obtained from the mode matching results are actually different for even and odd excitation. However, it has been found that this is only noticeable for relatively wide slits, where, in any case, the circuit model is not expected to be accurate. Thus, our conclusion is that developing a very fine model for thin screens is not practical. The model for the thick screens presented here can be used without significant errors if the slit width is not too large to preclude its applicability. 

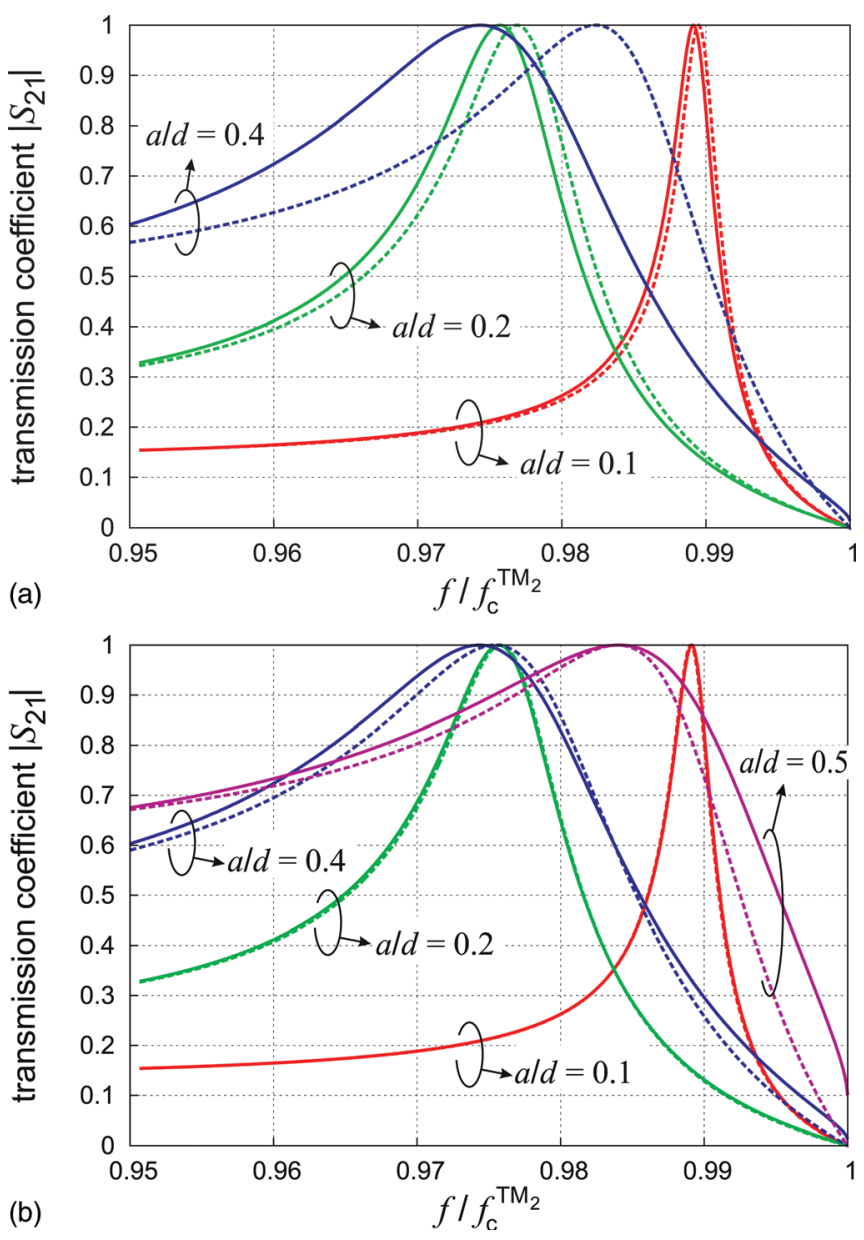

FIG. 5. (Color online) A closer look at the transmission spectra in Fig. 4 for frequencies close to the onset of the first grating lobe (extraordinary transmission region). The top figure uses analytical formulas for edge capacitances, while the bottom figure extracts these capacitances from a few full-wave data. The data corresponding to $a / d=0.5$, which were not present in Fig. 4, are also shown.

\section{THE LOSSY CONDUCTOR CASE}

Let us now examine the modifications required to introduce the material losses (i.e., the finite value of the conductivity) in our model. Most of the discussions reported in the previous section which are valid for perfect conductors, are also valid for lossy conductors. However, some important details must be revised. The main effect of having a real conductor (up to mid- infrared frequencies in the case of metals) is that magnetic fields and currents inside the conductor might not be negligible at all. For good conductors, such as metals at frequencies well below the optical regime, the electric field inside the conducting region is negligible (its energy is negligible, to be more precise), while the magnetic field is important and exponentially penetrates within the metal region along distances on the order of the skin penetration depth, $\delta_{s}=\sqrt{2 /\left(\omega \mu_{0} \sigma\right)}$ ( $\sigma$ is the real conductivity of the good conductor and $\mu_{0}$ is the permeability of vacuum). The magnetic field inside the conductor region was not present in the perfect conductor case. This means that the inductance per unit length of the parallel plate transmission line corresponding to the slit region is larger when real (not perfect) conductors are considered. The inductance associated with the magnetic field in the air region, $L_{2}=\left(\mu_{0} \varepsilon_{0}\right) / C_{2}=\mu_{0} a / W$, must be augmented by the socalled internal or incremental inductance, $\Delta L$. This incremental value is related to the skin effect resistance, $R_{s}$. For our simple parallel plate transmission lines, analytical formulas are available for those parameters: the skin effect resistance per unit length is $R_{s}=2 /\left(\delta_{s} W\right)$, and the incremental inductance is $\Delta L=R_{s} / \omega$.

The resistance, $R_{s}$, is responsible for the power loss that was not considered in the perfect conductor model. Power dissipation is the main effect for typical dimensions encountered in microwave practice, when the skin depth is much smaller than any other linear dimension of the structure. In such a case, the resistance, $R_{s}$, is retained in the transmission line model to account for attenuation, but the incremental inductance is neglected (in our case this is equivalent to consider, $\left.\Delta L \ll L_{2}\right)$. Under these assumptions, the wavenumber in the transmission line that models the slit region, $k$, becomes complex, but its real part is not very different from the propagation constant in the absence of losses, $k_{0}=\omega / c$. The imaginary part, i.e., the exponential attenuation factor, is small but significant. This approach is usually referred to as small losses approximation ${ }^{32}$ in transmission line theory. Our hypothesis is that the introduction of the per unit length resistance in the transmission line model accounting for the slit region is enough to accurately predict dissipation and reflection losses in a grating made with a nonperfect conductor. However, some authors have reported experimental results for slit systems where the skin depth is not negligible in comparison with the slit width, specifically for the case of a single slit. ${ }^{29}$ For a given screen thickness, $h$, the resonance frequencies monotonically increase when the slit width, $a$, is decreased, provided that perfect conductors are considered. The reason is that $\Delta h$ tends to vanish in the extremely narrow slit limit. Actually, this behavior is also observed in the case of lossy metallic conductors for relatively wide slits (more precisely, for slits having $a \gg \delta_{s}$ ). However, for a single slit it has been shown ${ }^{29}$ that this trend of the resonance frequencies drastically changes when $a$ is very small and the skin depth is not negligible. It will be shown here that this anomalous behavior also appears in periodic slit systems and can easily be accommodated in our model just by taking into account the effect of the internal inductance (in addition to the per unit length resistance).

Let us compute the complex propagation constant of the quasi-TEM mode supported by the slit region (note that strictly speaking, no TEM waves are allowed within the slit region if the conductor is lossy) using the general expression

$$
k^{2}=\left[\omega\left(L_{2}+\Delta L\right)-\mathrm{j} R_{s}\right] \omega C_{2},
$$

which is rigorous, and does not make any approximations. If $\Delta L$ is not negligible when compared to $L_{2}$, the phase constant of the quasi-TEM wave could be appreciably larger than the phase constant for lossless structures. Thus, the wavelength would be smaller than the free space wavelength. This effect is essential in order to account for the anomalous behavior of the FP resonances ${ }^{29}$ for very narrow slits. It is worth 
mentioning that the characteristic admittance also becomes complex and is given by

$$
Y_{2}=\sqrt{\frac{\omega C_{2}}{\omega\left(L_{2}+\Delta L\right)-\mathrm{j} R_{s}}} .
$$

Nevertheless, the formulas (15a), (15b), and (16) are still valid if complex arguments are used in the tangent and cotangent functions. It is obvious that the relevance of retaining the actual value of the internal inductance in the determination of the propagation constant and characteristic admittance of the slit region is more important when the slit width, $a$, is very small. The internal inductance might even be dominant if the slit width were smaller than skin depth, thus making the wavelength of the quasi-TEM wave propagating along the slit quite different from the free space wavelength at the operation frequency.

The losses over the metal walls outside the slits should also be accounted for in order to be completely rigorous. This could be done by adding a series resistance at the point of confluence of the high characteristic impedance transmission line section with the edge capacitance. However, the correction is very small because the dominant losses occur inside the slits. This resistance may have some importance when operating near the onset of the first grating lobe because, in such a case, a strong field enhancement occurs over that surface. However, it must be said that the skin effect resistance obtained by assuming a uniform current distribution along the $y$ direction would not be completely correct. In such a situation the electromagnetic field is dominated by the first higher order TM mode, whose field distribution along the $y$ direction is not uniform. Nevertheless, the order of magnitude of the resistance would be correct and a reasonable estimation of losses is expected.

\section{RESULTS FOR LOSSY GRATINGS}

Let us start this section checking the accuracy of our simple model for lossy screens with a few examples. In Fig. 6 the first order FP resonance through a periodic array of slits

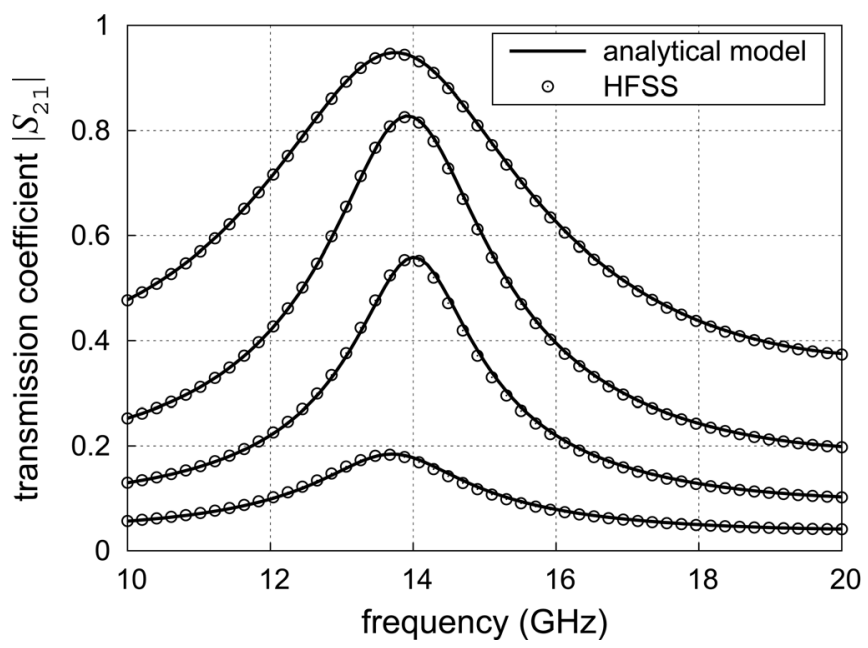

FIG. 6. First order Fabry-Pérot resonance of the transmission spectrum of an array of parallel slits in a lossy conducting medium $\left(\sigma=10^{5} \mathrm{~S} / \mathrm{m}\right)$. Four different values of slit width $(a=1.0,0.5,0.25$, and $0.1 \mathrm{~mm})$ are considered. Structure dimensions are $d=5.0 \mathrm{~mm}, h=10 \mathrm{~mm}$, and $W=1 \mathrm{~m}$. having four different widths is presented. The conductivity has been chosen small in comparison with metal conductivities $\left(\sigma=10^{5} \mathrm{~S} / \mathrm{m}\right)$ in order to enhance the effect of losses. Circles are full-wave data generated using the HFSs finite elements code, and solid lines correspond to the analytical model proposed in this paper. The agreement between analytical and numerical results is remarkable. It indicates that although losses outside the slit region have been neglected, the model gives a very good description of the transmissivity. It can be observed that the transmission level at resonance decreases when the slit with is reduced, as we expected. This reduction of transmission level is partially due to ohmic losses (absorption) but mismatching (reflected power) is also important. This can be clearly seen in Fig. 7, where transmission, reflection, and absorption are depicted for one of the cases in Fig. 6. Note that $70 \%$ of the incident power is not transmitted because about $50 \%$ is absorbed in the screen and $20 \%$ is reflected due to mismatching induced by losses (perfect matching would be expected for infinite conductivity). The proposed model works pretty well for arbitrary values of $a$ provided they are not too large. Thus, in Fig. 8 we can see the amplitude of the transmission coefficient at the first FP resonance frequency as a function of $a$. More than $50 \%$ of the impinging power is transmitted for slits wider than about $a=350 \mu \mathrm{m}$. For narrower slits the percentage of the incident power that is transmitted through the screen very quickly decreases. The slope of the curve in Fig. 8 becomes relatively large when the slit width is smaller than, roughly speaking, 20 times the skin depth (around 15 microns in this example).

The close inspection of the transmission curves in Fig. 6 shows us that the maximum transmission frequency for a given value of $h$ slightly depends on the slit width, $a$. Starting from the wider slit, a slight blueshift can be appreciated when the slit width is decreased. The main reason that explains this slight shift is that the excess length is smaller for narrower slits. Actually, in the absence of losses $(\sigma=\infty)$, the maximum transmission frequency should reach

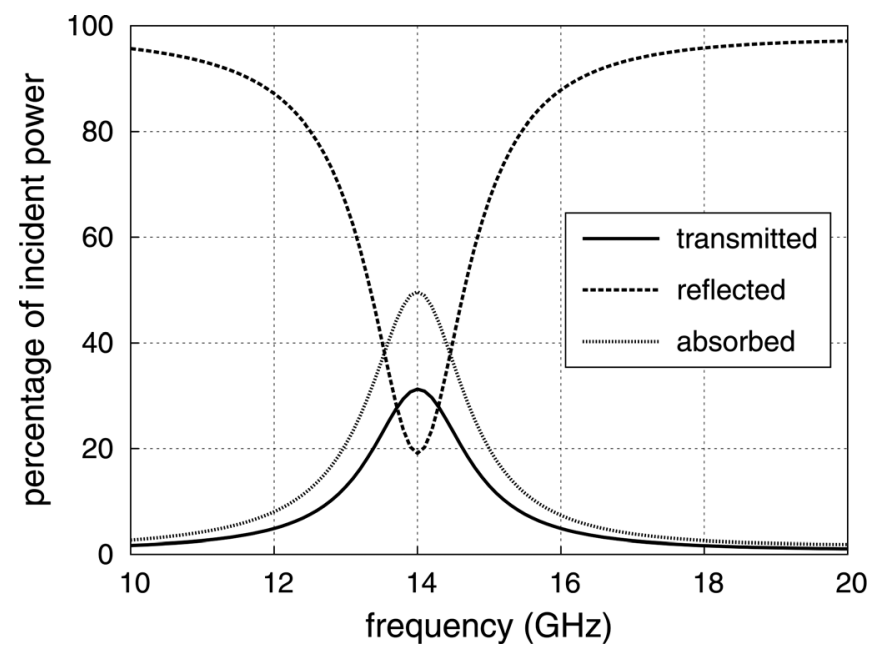

FIG. 7. Transmitted, reflected, and absorbed power as a function of frequency around the first order Fabry-Pérot resonance. Dimensions: $d=5.0 \mathrm{~mm}, h=10 \mathrm{~mm}, W=1 \mathrm{~m}$, and $a=0.25 \mathrm{~mm}$. Conductivity: $\sigma=10^{5} \mathrm{~S} / \mathrm{m}$. 


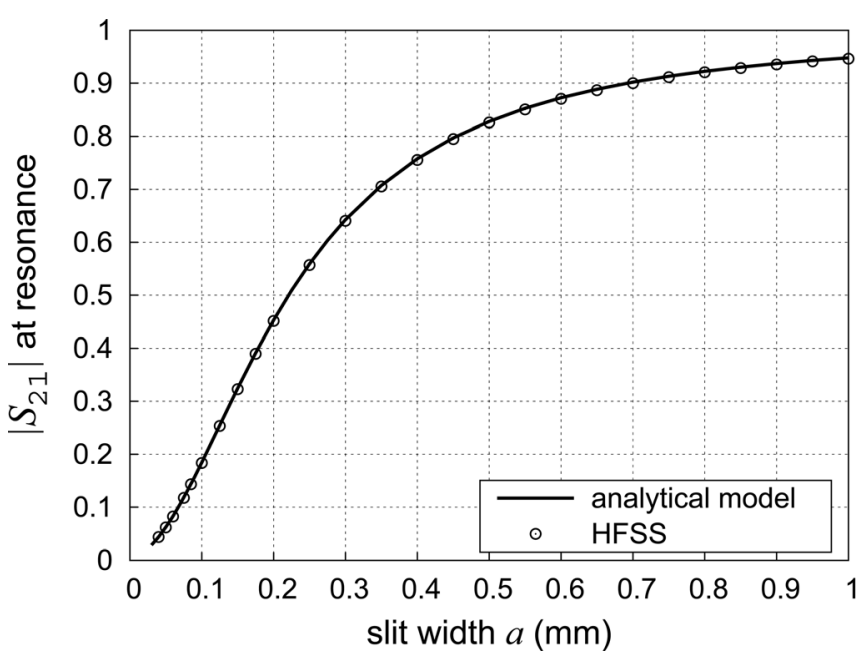

FIG. 8. Magnitude of the transmission coefficient as a function of the slit width. Structure dimensions are $d=5.0 \mathrm{~mm}, h=10 \mathrm{~mm}, W=1 \mathrm{~m}$, and $\sigma=10^{5} \mathrm{~S} / \mathrm{m}$.

the asymptotic value $c /(2 h)(14.99 \mathrm{GHz}$ for $h=10 \mathrm{~mm})$ in the very narrow slit limit. However the last curve (the one corresponding to the smallest slit width) does not follow this tendency. This phenomenon resembles the anomalous redshift reported for a single slit problem some years ago. ${ }^{29} \mathrm{We}$ have used our analytical model and the finite elements commercial simulator Ansoft HFSs to compute the frequencies of maximum transmission (first FP resonance) as a function of the slit width, for both perfect conducting and highly lossy screens. The results are reported in Fig. 9. The first observation is that, once again, the simple analytical model in this paper agrees remarkably well with the numerical data. For the perfect conductor grating it is clear from both the numerical and the analytical results that the FP resonance frequency increases monotonically when the slit width decreases. This is the expected behavior from the analysis of the single slit problem carried out in Ref. 12, for instance. Note that the FP resonance frequency tends to the theoretical limit (14.99 GHz) when a/d approaches zero. However,

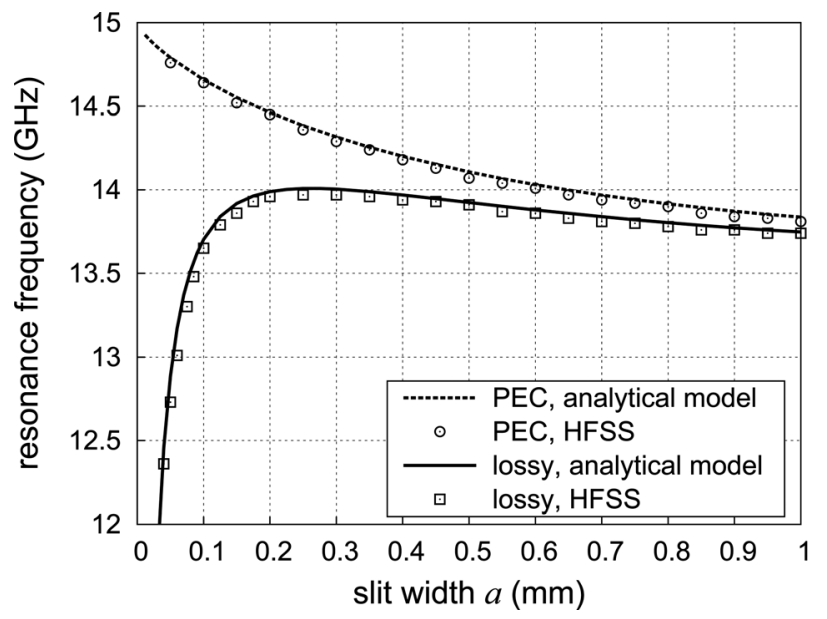

FIG. 9. Dependence of the first Fabry-Pérot resonance frequency with the slit width ( $a$ in Fig. 1) for a structure with period, $d=5 \mathrm{~mm}$, and screen thickness, $h=10.0 \mathrm{~mm}$. The perfect electric conductor (PEC) and the lossy conductor $\left(\sigma=10^{5} \mathrm{~S} / \mathrm{m}\right)$ cases are compared. Different behavior is observed in the narrow slit limit. when a finite conductivity is used to model the conducting screen, analytical and numerical results predict a different behavior. For relatively wide slits the behavior is the same observed for perfect conductors, with a slight additional redshift of the former that can be explained in terms of the influence of a small internal inductance in the conducting material; as explained above, the real part of the complex wavenumber is slightly larger for lossy parallel plate waveguides than for lossless structures. Nevertheless, the influence on the FP transmission frequency of the edge effect is dominant (the edge effect is considered to be the same for lossless and lossy materials since the electric field inside the conductors is negligible). Thus, an increase of FP resonance frequency is expected, even in the presence of losses, when the slit width is reduced in size. However, it is observed in Fig. 9 that this tendency is broken in the lossy case below 250 microns. This size is about 20 times the skin depth in the conducting material considered here at the FP resonance frequency. Below that threshold the effect of internal inductance on the quasi-TEM mode propagation constant governs the behavior of the evolution of the FP resonance frequency with respect to the $a$ parameter. Edge capacitance favors an increase of the resonance frequency, but the dominant effect of the internal inductance prevails and the final result is a quick reduction of the resonance frequency. This effect is accompanied by strong absorption and mismatching, of course. This behavior is qualitatively identical to the behavior of a single slit reported in Fig. 2 of Ref. 29, however, quantitatively the shift is strongly affected by the period of the structure (this parameter is obviously absent in the single slit problem).

Finally, it is worth mentioning that the theory reported up to this point remains valid for slits filled with dielectric materials. The characteristic admittance of the slit region should be changed accordingly by multiplying the free space admittance by the square root of the relative permittivity. However, no closed-form expression would be available for the edge capacitance, although an approximate formula could be derived using the same conformal mapping used for the free space case. Dielectric losses could be trivially incorporated by using a shunt conductance in the transmission line model. ${ }^{32}$

\section{CONCLUSION}

Resonant transmission of microwaves or millimeter waves through diffraction gratings made of periodically distributed and closely spaced lossy conducting bars has been analyzed using a very simple and analytical model. Relying on common microwave engineering methods, the model leads to an equivalent circuit whose parameters are known in closed form. Physical insight into the frequency shift of Fabry-Pérot-like behavior has been gained using the concept of edge capacitance and equivalent thickness. The anomalous behavior of the resonance frequencies with respect to the slit width in the very narrow slit limit has been explained by including the effect of the internal inductance of the lossy conductors in the model. In general, the model accounts very accurately for the effect of losses even though some sources 
of losses are neglected. The model qualitatively explains the shape and main features of the transmission spectrum and provides a quantitative method to obtain the transmissivity at any frequency below the onset of the first grating lobe.

\section{ACKNOWLEDGMENTS}

Dr. Rui Yang's work has been supported by Newton International Fellowships from the Royal Society, U.K., 2009. F. Medina's work has been carried out during his stay at the Dept. of Electronic Engineering of the QMUL (London, UK), funded by the Spanish Ministry of Science and Innovation, Mobility Program, Grant No. P2009-0405. R. Rodríguez-Berral and F. Medina also thank the support of the Spanish Ministry of Science and Innovation and European Union FEDER funds (Project Nos. TEC2007-65376, TEC2010-16948, and Consolider-Ingenio 2010 Project No. CSD2008-00066), and the Spanish Junta de Andaluca (Project No. TIC-4595).

${ }^{1}$ T. W. Ebbesen, H. J. Lezec, H. F. Ghaemi, T. Thio, and P. A. Wolff, Nature (London) 391, 667 (1998).

${ }^{2}$ C. Genet and T.W. Ebbesen, Nature (London) 445, 39 (2007).

${ }^{3}$ F. J. García-de-Abajo, Rev. Mod. Phys. 79, 1267 (2007).

${ }^{4}$ F. J. García-Vidal, L. Martín-Moreno, T. W. Ebbesen, and L. Kuipers, Rev. Mod. Phys. 82, 729 (2010).

${ }^{5}$ R. Gordon, A. G. Brolo, D. Sinton, and K. L. Kavanagh, Laser Photonics Rev. 4, 311 (2010).

${ }^{6}$ J. A. Porto, F. J. García-Vidal, and J. B. Pendry, Phys. Rev. Lett. 83, 2845 (1999).

${ }^{7}$ V. G. Sologub, V. P. Schestopalov, and G. G. Polovnikov, J. Tech. Phys. 37, 667 (1967) (in Russian).

${ }^{8}$ S. Astilean, P. Lalanne, and M. Palamaru, Opt. Commun. 175, 265 (1967).

${ }^{9}$ H. E. Went, A. P. Hibbins, J. R. Sambles, C. R. Lawrence, and A. P. Crick, Appl. Phys. Lett. 77, 2789 (2000).
${ }^{10}$ D. C. Skigin and R. A. Depine, Phys. Rev. Lett. 95, 217402 (2000).

${ }^{11}$ D. C. Skigin and R. A. Depine, Phys. Rev. E 74, 046606 (2000).

${ }^{12}$ Y. Takakura, Phys. Rev. Lett. 86, 5601 (2001).

${ }^{13}$ F. Yang and J. R. Sambles, Phys. Rev. Lett. 89, 063901 (2002).

${ }^{14}$ Y. Qi, J. Miao, S. Hong, and M. M. Tentzeris, IEEE Trans. Microwave Theory Tech. 58, 3657 (2010).

${ }^{15}$ L. A. Dunbar, M. Guillaumée, F. de León-Pérez, C. S. E. Grenet, R. Eckert, F. López-Tejeira, F. J. García-Vidal, L. Martín-Moreno, and R. P. Stanley, Appl. Phys. Lett. 95, 011113 (2009).

${ }^{16}$ F. J. Valdivia-Valero and M. Nieto-Vesperinas, Opt. Express 18, 6740 (2010).

${ }^{17}$ W. Tang, Y. Hao, and F. Medina, Opt. Express 18, 16946 (2010).

${ }^{18}$ E. Moreno, L. Martín-Moreno, and F. J. García-Vidal, J. Opt. A, Pure Appl. Opt. 8, 94 (2006).

${ }^{19}$ N. G. Don, A. A. Kirilenko, and S. L. Senkevich, Radiophys. Quantum Electron. 51, 101 (2008).

${ }^{20}$ Y. Pang, A. N. Hone, P. P. M. So, and R. Gordon, Opt. Express 17, 4433 (2009).

${ }^{21}$ F. Medina, J. A. Ruiz-Cruz, F. Mesa, J. M. Rebollar, J. R. Montejo-Garai, and R. Marqués, Appl. Phys. Lett. 95, 071102 (2009).

${ }^{22}$ F. Medina, F. Mesa, J. A. Ruiz-Cruz, J. M. Rebollar, and J. R. MontejoGarai, IEEE Trans. Microwave Theory Tech. 58, 1532 (2010).

${ }^{23}$ M. Beruete, I. Campillo, M. Navarro-Cía, F. Falcone, and M. Sorolla, IEEE Trans. Antennas Propag. 55, 1514 (2007).

${ }^{24}$ F. Medina, F. Mesa, and R. Marqués, IEEE Trans. Microwave Theory Tech. 56, 3108 (2008).

${ }^{25}$ C.-M. Wang, Y.-C. Chang, and D. P. Tsai, Opt. Express 17, 6218 (2009).

${ }^{26}$ X. F. Li and S. F. Yua, J. Appl. Phys. 108, 013302 (2010).

${ }^{27}$ X.-R. Huang, R.-W. Peng, and R.-H. Fan, Phys. Rev. Lett. 105, 243901 (2010).

${ }^{28}$ F. Medina, F. Mesa, and D. C. Skigin, IEEE Trans. Microwave Theory Tech. 58, 105 (2010).

${ }^{29}$ J. R. Suckling, A. P. Hibbins, M. J. Lockyear, T. W. Preist, J. R. Sambles, and C. R. Lawrence, Phys. Rev. Lett. 92, 147401 (2004).

${ }^{30}$ X. Wang, Appl. Phys. Lett. 96, 134104 (2010).

${ }^{31}$ J. D. Jackson, Classical Electrodynamics, 3rd ed. (John Wiley \& Sons, New York, 1998).

${ }^{32}$ R. E. Collin, Field Theory of Guided Waves, 2nd ed. (IEEE Press, Piscataway, New Jersey, 1991). 